\title{
Small dilatation mapping classes coming from the simplest hyperbolic braid
}

\author{
ERIKO HIRONAKA
}

In this paper we study the small dilatation pseudo-Anosov mapping classes arising from fibrations over the circle of a single 3-manifold, the mapping torus for the "simplest hyperbolic braid". The dilatations that occur include the minimum dilatations for orientable pseudo-Anosov mapping classes for genus $g=2,3,4,5$ and 8 . We obtain the "Lehmer example" in genus $g=5$, and Lanneau and Thiffeault's conjectural minima in the orientable case for all genus $g$ satisfying $g=2$ or $4(\bmod 6)$. Our examples show that the minimum dilatation for orientable mapping classes is strictly greater than the minimum dilatation for non-orientable ones when $g=4,6$ or 8 . We also prove that if $\delta_{g}$ is the minimum dilatation of pseudo-Anosov mapping classes on a genus $g$ surface, then

$$
\limsup _{g \rightarrow \infty}\left(\delta_{g}\right)^{g} \leq \frac{3+\sqrt{5}}{2} .
$$

57M50; 57M25

\section{Introduction}

Let $S_{g}$ be a closed oriented surface of genus $g \geq 1$, and let $\operatorname{Mod}_{g}$ be the mapping class group of isotopy classes of orientation preserving self-homeomorphisms of $S_{g}$. A mapping class $\phi \in \operatorname{Mod}_{g}$ is called pseudo-Anosov if $S_{g}$ admits a pair of $\phi$-invariant, transverse measured, singular foliations on which $\phi$ acts by stretching transverse to one foliation by a constant $\lambda(\phi)>1$ and contracting transverse to the other by $\lambda(\phi)^{-1}$. The constant $\lambda(\phi)$ is called the (geometric) dilatation of $\phi$. A mapping class is pseudoAnosov if it is neither periodic nor reducible (see Thurston [26], Fathi, Laudenbach and Poenaru [7], and Casson and Bleiler [3]). Denote by $\operatorname{Mod}_{g}^{\mathrm{pA}}$ the set of pseudo-Anosov mapping classes in $\operatorname{Mod}_{g}$.

A pseudo-Anosov mapping class $\phi$ is defined to be orientable if its invariant foliations are orientable. We will denote the set of orientable pseudo-Anosov mapping classes by $\operatorname{Mod}_{g}^{\mathrm{pA}+}$. Let $\lambda_{\text {hom }}(\phi)$ be the spectral radius of the action of $\phi$ on the first homology of $S$. Then

$$
\lambda_{\text {hom }}(\phi) \leq \lambda(\phi)
$$


with equality if and only if $\phi$ is orientable (see, for example, Lanneau and Thiffeault [16], and Koberda and Silberstein [15]).

The dilatations $\lambda(\phi)$ satisfy reciprocal monic integer polynomials of degree bounded from above by $6 g-6$ (see Thurston [26]). If $\phi$ is orientable the degree is bounded by $2 g$. For fixed $g$, it follows that $\lambda(\phi)$ achieves a minimum $\delta_{g}>1$ on $\operatorname{Mod}_{g}^{\mathrm{pA}}$ (see also, Arnoux and Yoccoz [2] and Ivanov [12]). Let

$$
\operatorname{Mod}_{g}^{\mathrm{pA}+} \subset \operatorname{Mod}_{g}^{\mathrm{pA}}
$$

be the subset of orientable pseudo-Anosov mapping classes, and let $\delta_{g}^{+}$be the minimum dilatation among elements of $\operatorname{Mod}_{g}^{\mathrm{pA}+}$.

In this paper, we address the following question (see Penner [23], McMullen [20] and Farb [5]):

Question 1.1 What is the behavior of $\delta_{g}$ and $\delta_{g}^{+}$as functions of $g$ ?

So far, exact values of $\delta_{g}$ have only been found for $g \leq 2$. For $g=1$, the derivative map determines an identification $\operatorname{Mod}_{1}=\operatorname{SL}(2 ; \mathbb{Z})$, and

$$
\delta_{1}=\frac{3+\sqrt{5}}{2} .
$$

For a monic integer polynomial $p(x)$, the house of $p(x)$, written $|p|$, is the absolute value of the largest root of $p$. For $g=2$, Cho and Ham [4] show that $\delta_{2}$ is given by

$$
\left|t^{4}-t^{3}-t^{2}-t+1\right| \approx 1.72208 .
$$

In the orientable case more is known due to recent results of Lanneau and Thiffeault [16]. Given $(a, b) \in \mathbb{Z} \oplus \mathbb{Z}$ with $0<a<b$, let

$$
L T_{(a, b)}(t)=t^{2 b}-t^{b}\left(1+t^{a}+t^{-a}\right)+1,
$$

and let

$$
\lambda_{(a, b)}=\left|L T_{(a, b)}(t)\right| .
$$

Theorem 1.2 (Lanneau-Thiffeault [16, Theorem 1.2 and 1.3]) For $g=2,3,4,6$ and 8 ,

$$
\lambda_{(1, g)} \leq \delta_{g}^{+}
$$

with equality when $g=2,3$ or 4 . 
For $g=2$, the value of $\delta_{2}^{+}$was first determined by Zhirov [27]. For $g=5$, Lanneau and Thiffeault show that $\delta_{5}^{+}$equals Lehmer's number $(\approx 1.17628)$ [17]. This dilatation is realized as a product of multi-twists along a curve arrangement dual to the $E_{10}$ Coxeter graph (see Leininger [18] and Hironaka [10]), and as the monodromy of the $(-2,3,7)$-pretzel knot (see Hironaka [9]). Lanneau and Thiffeault also find a lower bound for $\delta_{7}^{+}$. An example realizing this bound can be found in Aaber and Dunfield [1, page 4] and Kin and Takasawa [14, Theorem 1.12].

Based on their results, Lanneau and Thiffeault ask:

Question 1.3 (Lanneau-Thiffeault [16, Question 6.1]) Is $\delta_{g}^{+}=\lambda_{(1, g)}$ for all even $g$ ? For convenience, we will call the affirmative answer to their question the LT-conjecture. In our first result, we improve on the following previous best bounds for the minimum dilatation of infinite families

$$
\left(\delta_{g}\right)^{g} \leq\left(\delta_{g}^{+}\right)^{g} \leq 2+\sqrt{3}
$$

found in Minakawa [22], and Hironaka-Kin [11].

Theorem 1.4 If $g=0,1,3$ or $4(\bmod 6), g \geq 3$, then

$$
\delta_{g} \leq \lambda_{(3, g+1)}
$$

and if $g=2$ or $5(\bmod 6)$ and $g \geq 5$, then

$$
\delta_{g} \leq \lambda_{(1, g+1)} .
$$

For the orientable case, our results complement those of Lanneau and Thiffeault for $g=2$ or $4(\bmod 6)$.

Theorem 1.5 Let $g \geq 3$. Then

$$
\begin{array}{ll}
\delta_{g}^{+} \leq \lambda_{(3, g+1)} & \text { if } g=1 \text { or } 3(\bmod 6), \\
\delta_{g}^{+} \leq \lambda_{(1, g)} & \text { if } g=2 \text { or } 4(\bmod 6), \text { and } \\
\delta_{g}^{+} \leq \lambda_{(1, g+1)} & \text { if } g=5(\bmod 6) .
\end{array}
$$

Putting Theorem 1.5 together with Lanneau and Thiffeault's lower bound for $g=8$ gives:

Corollary 1.6 The minimal dilatation for orientable pseudo-Anosov mapping classes for genus 8 is given by

$$
\delta_{8}^{+}=\lambda_{(1,8)}
$$


The following is a table of the minimal dilatations that arise in this paper's examples for genus 1 through 12. All numbers in the table are truncated to 5 decimal places. An asterisk $*$ marks the numbers that have been verified to equal $\delta_{g}^{+}$(resp., $\delta_{g}$ ). For singularity-type, we use the convention that $\left(a_{1}, \ldots, a_{k}\right)$ means that the singularities of the invariant foliations have degrees $a_{1}, \ldots, a_{k}$ (see Lanneau and Thiffeault's notation [16, page 3]). The singularity-types for our examples are derived from the formula given in Corollary 3.6.

\begin{tabular}{|c||l|l||l|l|}
\hline$g$ & orientable & degrees of singularities & unconstrained & degrees of singularities \\
\hline 1 & $2.61803^{*}$ & no singularities & $2.61803^{*}$ & no singularities \\
\hline 2 & $1.72208^{*}$ & $(4)$ & $1.72208^{*}$ & $(4)$ \\
\hline 3 & $1.40127^{*}$ & $(2,2,2,2)$ & 1.40127 & $(2,2,2,2)$ \\
\hline 4 & $1.28064^{*}$ & $(10,2)$ & 1.26123 & $(3,3,3,3)$ \\
\hline 5 & $1.17628^{*}$ & $(16)$ & 1.17628 & $(16)$ \\
\hline 6 & - & - & 1.1617 & $(5,5,5,5)$ \\
\hline 7 & 1.13694 & $(6,6,6,6)$ & 1.13694 & $(6,6,6,6)$ \\
\hline 8 & $1.12876^{*}$ & $(22,6)$ & 1.1135 & $(25,1,1,1)$ \\
\hline 9 & 1.1054 & $(8.8 .8 .8)$ & 1.1054 & $(8,8,8,8)$ \\
\hline 10 & 1.10149 & $(28,8)$ & 1.09466 & $(9,9,9,9)$ \\
\hline 11 & 1.08377 & $(34,2,2,2)$ & 1.08377 & $(34,2,2,2)$ \\
\hline 12 & - & - & 1.07874 & $(11,11,11,11)$ \\
\hline
\end{tabular}

Table 1: Minimal orientable and unconstrained dilatations coming from $M_{\mathrm{sb}}$

For $g=2,3,4$ and 5, our orientable examples agree both in dilatation and in singularitytype with the previously known minimizing examples (see [16, Sections 3, 4 and 6]). For $g=8$, our example agrees with the singularity-type anticipated by Lanneau and Thiffeault $[16,(6.4)]$. We prove that the known minimal dilatation examples for $g=2,3,4,5$ and 8 arise as the monodromy of fibrations of a single 3-manifold $M_{\mathrm{sb}}$. For $g=7$, our minimal example gives a larger dilatation than $\delta_{7}^{+}$. (The dilatation $\delta_{7}^{+}$ is realized by Kin and Takasawa [14], and by Aaber and Dunfield [1].)

Lanneau and Thiffeault show that $\delta_{5}^{+} \leq \delta_{6}^{+}$, and hence $\delta_{g}^{+}$is not strictly monotone decreasing (see Farb [5, Question 7.2]). Theorem 1.5 implies the following stronger statement.

Proposition 1.7 If the LT-conjecture is true, then $\delta_{g}^{+} \leq \delta_{g+1}^{+}$, whenever $g=5(\bmod 6)$. 
Another consequence concerns the question of when the inequality $\delta_{g} \leq \delta_{g}^{+}$is strict. In [14] and [1] it is shown that $\delta_{5}<\delta_{5}^{+}$. Table 1 shows the following.

Corollary 1.8 For $g=4,6$ and 8 we have

$$
\delta_{g}<\delta_{g}^{+} .
$$

Theorem 1.4 and Proposition 4.3 imply the following.

Proposition 1.9 If the LT-conjecture is true, then for all even $g \geq 4$ we have

$$
\delta_{g}<\delta_{g}^{+} .
$$

For large $g$, it is known that $\delta_{g}$ and $\delta_{g}^{+}$converges to 1 . Furthermore,

$$
\log \left(\delta_{g}\right) \asymp \frac{1}{g} \quad \text { and } \quad \log \left(\delta_{g}^{+}\right) \asymp \frac{1}{g}
$$

(see Penner [23], McMullen [20], Minakawa [22] and Hironaka-Kin [11]). The LTconjecture together with (1) leads to the natural question:

Question 1.10 (See McMullen [20, page 551], Farb [5, Problem 7.1]) Do the sequences

$$
\left(\delta_{g}\right)^{g} \quad \text { and } \quad\left(\delta_{g}^{+}\right)^{g}
$$

converge as $g$ grows? What is the limit?

Theorem 1.4 and Theorem 1.5 imply the following.

\section{Theorem 1.11}

$$
\limsup _{g \rightarrow \infty}\left(\delta_{g}\right)^{g} \leq \frac{3+\sqrt{5}}{2} \quad \text { and } \quad \limsup _{g \neq 0(\bmod 6)}\left(\delta_{g}^{+}\right)^{g} \leq \frac{3+\sqrt{5}}{2} .
$$

This leads to the question:

Question 1.12 (Golden Mean Question) Do the sequences $\left(\delta_{g}\right)^{g}$ and $\left(\delta_{g}^{+}\right)^{g}$ satisfy

$$
\lim _{g \rightarrow \infty}\left(\delta_{g}\right)^{g}=\lim _{g \rightarrow \infty}\left(\delta_{g}^{+}\right)^{g}=\frac{3+\sqrt{5}}{2}=(\text { golden mean })^{2} ?
$$


For any pseudo-Anosov mapping class $\phi$, let $M(\phi)$ be the mapping torus of $\phi$. Conversely, given a compact hyperbolic 3-manifold with torus boundary components $M$, let $\Phi(M)$ be the collection of pseudo-Anosov mapping classes $\phi$ such that $M=M(\phi)$. Let $\Sigma$ be the suspensions of singularities of the stable and unstable foliations of $\phi$ and let

$$
M^{*}(\phi)=M(\phi) \backslash \Sigma
$$

Theorem 1.13 (Farb, Leininger and Margalit [6, Theorem 1.1]) The set

$$
\mathcal{T}_{P}=\left\{M^{*}(\phi): \phi \in \operatorname{Mod}_{g}^{\mathrm{pA}}, \lambda(\phi) \leq P^{1 / g}\right\}
$$

is finite for any $P>1$.

The asymptotic equations (1) and Theorem 1.13 imply that

$$
\begin{aligned}
\mathcal{T} & =\left\{M^{*}(\phi): \phi \in \operatorname{Mod}_{g}^{\mathrm{pA}}, \lambda(\phi)=\delta_{g}\right\} \\
\text { and } \quad \mathcal{T}^{+} & =\left\{M^{*}(\phi): \phi \in \operatorname{Mod}_{g}^{\mathrm{pA}+} \lambda(\phi)=\delta_{g}^{+}\right\}
\end{aligned}
$$

are finite.

This leads to the question:

Question 1.14 How large are the sets $\mathcal{T}$ and $\mathcal{T}^{+}$?

If the LT-conjecture is true, then our results imply that a single 3-manifold $M_{\mathrm{sb}}$ would realize $\delta_{g}^{+}$for all $g=2,4(\bmod 6)$. The manifold $M_{\mathrm{sb}}$ is the complement of the $6_{2}^{2}$ braid (see Rolfsen's tables [24], and Figure 1). Another 3-manifold that produces small dilatation mapping classes is the complement $M_{-2,3,8}$ of the $(-2,3,8)$-pretzel link in $S^{3}$. These have been studied independently by Kin and Takasawa [14] and by Aaber and Dunfield [1]. For certain genera the mapping classes in $\Phi\left(M_{-2,3,8}\right)$ have smaller dilatation than the minima realized by $M_{\mathrm{sb}}$, but the asymptotic behavior of the minimal dilatations for large genus, supports the affirmative to Question 1.12. Both $M_{-2,3,8}$ and $M_{\mathrm{sb}}$ can be obtained from the magic manifold by Dehn fillings (see Martelli and Petronio [19]). The pseudo-Anosov braid monodromies with smallest known dilatations found in Hironaka-Kin [11] are also realized on the magic manifold (see Kin-Takasawa [13]).

Section 2 contains a brief review of Thurston norms, fibered faces and the Teichmüller polynomial. These are the basic tools used in this paper. In Section 3 we describe our family of examples, and in Section 4 we prove Theorem 1.4 and Theorem 1.5. 
Acknowledgments The author thanks C McMullen and T Koberda for many helpful conversations, and E Kin, S Dowdall and the referee of this article for comments and corrections to earlier versions. The author is grateful for the hospitality of the Harvard Mathematics Department, where she wrote this paper as a visiting scholar in Fall 2009.

\section{Background and tools}

In this section we give a brief review of invariants and properties of fibrations of a hyperbolic 3-manifold $M$, emphasizing the tools that we will use in the rest of the paper. For more details see, for example, Thurston [25], Fathi-Laudenbach-Poenaru [7], and McMullen [20; 21].

The theory of fibered faces of the Thurston norm ball and the existence of Teichmüller polynomials provides a way to study in a single picture a collection of pseudo-Anosov mapping classes defined on surfaces of different Euler characteristics and genera. Assume $M$ is a compact hyperbolic 3-manifold with boundary. Given an embedded orientable surface $S$ on $M$, let $\chi_{-}(S)$ be the sum of $\left|\chi\left(S_{i}\right)\right|$, where $S_{i}$ are the connected components of $S$ with negative Euler characteristic. The Thurston norm of $\psi \in \mathrm{H}^{1}(M ; \mathbb{Z})$ is defined to be

$$
\|\psi\|_{T}=\min \chi_{-}(S),
$$

where the minimum is taken over oriented embedded surfaces $(S, \partial S) \subset(M, \partial M)$ such that the class of $(S, \partial S)$ in $\mathrm{H}_{2}(M, \partial M ; \mathbb{Z})$ is the Poincare dual of $\psi$.

Elements of $\mathrm{H}^{1}(M ; \mathbb{Z})$ are canonically associated with epimorphisms

$$
\pi_{1}(M ; \mathbb{Z}) \rightarrow \mathbb{Z}
$$

We thus make the following natural identification:

$$
\mathrm{H}^{1}(M ; \mathbb{Z})=\operatorname{Hom}\left(\pi_{1}(M), \mathbb{Z}\right)=\operatorname{Hom}\left(\mathrm{H}_{1}(M ; \mathbb{Z}), \mathbb{Z}\right)
$$

We consider this as a lattice $\Lambda_{M}$ inside $\mathbb{R}^{b_{1}(M)}$, where $b_{1}(M)$ is the first Betti number of $M$. If $\psi \in \Lambda_{M}$ corresponds to a fibration

$$
\psi: M \rightarrow S^{1}
$$

we say that $\psi$ is fibered. In this case the Thurston norm of $\psi$ is given by

$$
\|\psi\|_{T}=\chi_{-}(S)
$$

where $S$ is homeomorphic to the fiber of $\psi$. Let

$$
\Psi(M)=\left\{\psi: M \rightarrow S^{1}: \psi \text { is a fibration }\right\} .
$$


The monodromy $\phi$ of $\psi \in \Psi(M)$ is the mapping class $\phi: S \rightarrow S$, such that $M$ is the mapping torus of $\phi$, and $\psi$ is the natural projection to $S^{1}$. Since $M$ is hyperbolic, $\phi$ is pseudo-Anosov.

Let $B$ be the unit ball in $\mathbb{R}^{b_{1}(M)}$ with respect to the extended Thurston norm.

Theorem 2.1 (Thurston [25]) The Thurston norm ball $B$ is a convex polyhedron and for any top-dimensional open face $F$ of $B,\left(F \cdot \mathbb{R}^{+}\right) \cap \Psi(M)$ is either empty or equal to $\left(F \cdot \mathbb{R}^{+}\right) \cap \Lambda_{M}$.

If $\left(F \cdot \mathbb{R}^{+}\right) \cap \Psi(M) \neq \varnothing$, we say $F$ is a fibered face of $B$. An element of $\Psi(M)$ is called primitive if its fiber is connected. The elements of $\Lambda_{M}$ project to the rational points on the boundary of $B$. If $F$ is a fibered face, then each rational point $x$ on $F$ corresponds to a unique primitive element $\psi_{x} \in \Psi(M)$, namely the element of $\left(x \cdot \mathbb{R}^{+}\right) \cap \Psi(M)$ that lies closest to the origin.

Theorem 2.2 (Fried [8, Theorem E]) There is a continuous function $\mathcal{Y}$, homogeneous of degree one, defined on the fibered cone in $\mathbb{R}^{b_{1}(M)}$, so that if $\psi$ is fibered with monodromy $\phi_{\psi}$, then

$$
\mathcal{Y}(\psi)=\frac{1}{\log \left(\lambda\left(\phi_{\psi}\right)\right)} .
$$

The function $\mathcal{Y}$ is concave and tends to zero along the boundary of the cone.

Corollary 2.3 For each fibered face $F$,

$$
\bar{\lambda}(\psi)=\lambda\left(\phi_{\psi}\right)^{\|\psi\|_{T}},
$$

extends to a continuous function on $F \cdot \mathbb{R}^{+}$that is constant on rays through the origin, and $\bar{\lambda}$ achieves a unique minimum on $F$.

Let $G$ be a group and $\psi: G \rightarrow \mathbb{Z}$ a homomorphism. If $f \in \mathbb{Z}[G]$ is given by

$$
f=\sum_{g \in G} \alpha_{g} g
$$

then the specialization of $f$ at $\psi$ is the polynomial in $\mathbb{Z}[t]$ defined by

$$
f^{\psi}(t)=\sum_{g \in G} \alpha_{g} t^{\psi(g)}
$$


Theorem 2.4 (McMullen [20]) Let $F$ be a fibered face for a 3-manifold $M$, and let $G=H_{1}(M ; \mathbb{Z})$. Then there is an element $\theta_{F} \in \mathbb{Z}[G]$ such that for all integral lattice points $\psi$ in the fibered cone of $F$,

$$
\lambda\left(\phi_{\psi}\right)=\left|\theta_{F}^{\psi}\right|
$$

The polynomial $\theta_{F}$ is called the Teichmüller polynomial of $M$ for the fibered face $F$.

\section{The mapping torus for the simplest hyperbolic braid}

We now look at a particular 3-manifold, and study properties of its fibrations. This example has also been studied by McMullen [20, Section 11], and the first part of this section will be a review of what is found there.
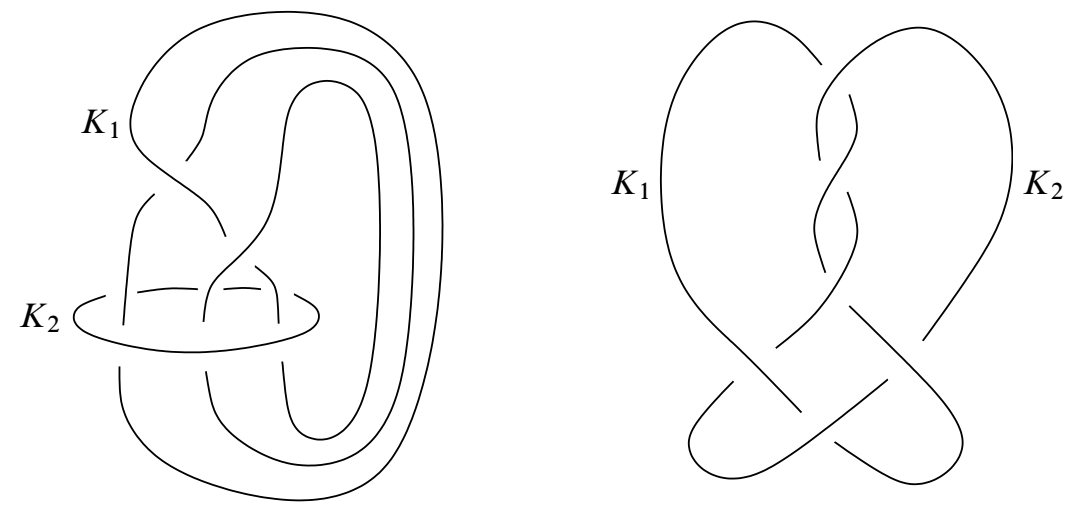

Figure 1: Two diagrams for the link $6_{2}^{2}$

Let $M=S^{3} \backslash N(L)$, where $L$ is the link drawn in two ways in Figure 1, and $N(L)$ is a tubular neighborhood. As seen from the left diagram in Figure 1, $M$ fibers over the circle with fiber a sphere with four boundary components $S_{0,4}$. Let $\psi_{0}: M \rightarrow S^{1}$ be the corresponding fibration, and let $\phi_{0}: S_{0,4} \rightarrow S_{0,4}$ be the monodromy. Then $\phi_{0}$ is the mapping class associated to the braid written with respect to standard generators as $\sigma_{1} \sigma_{2}^{-1}$ (see Figure 2) and its dilatation is given by

$$
\lambda\left(\phi_{0}\right)=\frac{3+\sqrt{5}}{2} .
$$

The braid $\sigma_{1} \sigma_{2}^{-1}$ has been called the "simplest hyperbolic braid" (see McMullen [20, Section 11]). 


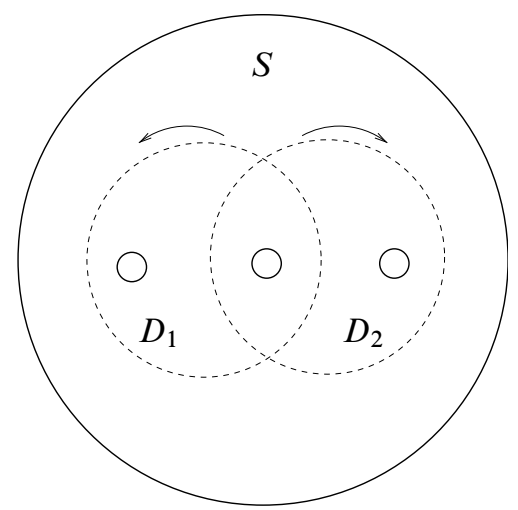

Figure 2: Braid monodromy associated to $\sigma_{1} \sigma_{2}^{-1}$

Let $K_{1}$ and $K_{2}$ be the components of $L$ as drawn in Figure 1. Let $\mu_{1}$ be the meridian of $K_{1}$ and $\mu_{2}$ be the meridian of $K_{2}$. These determine coordinate functions for $H^{1}(M ; \mathbb{Z})$

$$
\left(\mu_{1}, \mu_{2}\right)(\psi)=\left(\psi\left(\mu_{1}\right), \psi\left(\mu_{2}\right)\right) \in \mathbb{Z} \times \mathbb{Z} .
$$

With respect to these coordinates, the Thurston norm and the Alexander norm both are given by

$$
\|(a, b)\|=\max \{2|a|, 2|b|\} .
$$

The lattice points $\Lambda_{M}$ in the fibered cone $F \cdot \mathbb{R}^{+}$defined by $\psi=(0,1)$ is the set

$$
\Psi=\{(a, b) \in \mathbb{Z} \times \mathbb{Z}: b>0,-b<a<b\}
$$

as shown in Figure 3. For the rest of this paper, we will only be concerned with the subset $\Psi_{\text {prim }} \subset \Psi$ consisting of elements of $\Psi$ with connected fibers, i.e., the primitive elements. Thus,

$$
\Psi_{\text {prim }}=\{(a, b) \in \mathbb{Z} \times \mathbb{Z}: b>0,-b<a<b, \operatorname{gcd}(a, b)=1\} .
$$

The Alexander polynomial for $L$ is given by

$$
\Delta_{L}(x, u)=u^{2}-u\left(1-x-x^{-1}\right)+1
$$

(see Rolfsen's table [24]), and the Teichmüller polynomial is given by

$$
\Theta_{L}(x, u)=u^{2}-u\left(1+x+x^{-1}\right)+1
$$

(see [20, page 47]).

Specialization to the element $(a, b) \in \mathrm{H}^{1}(M ; \mathbb{Z})$ is the same as plugging $\left(t^{a}, t^{b}\right)$ into the equations for the Alexander and Teichmüller polynomials (see Section 2). 


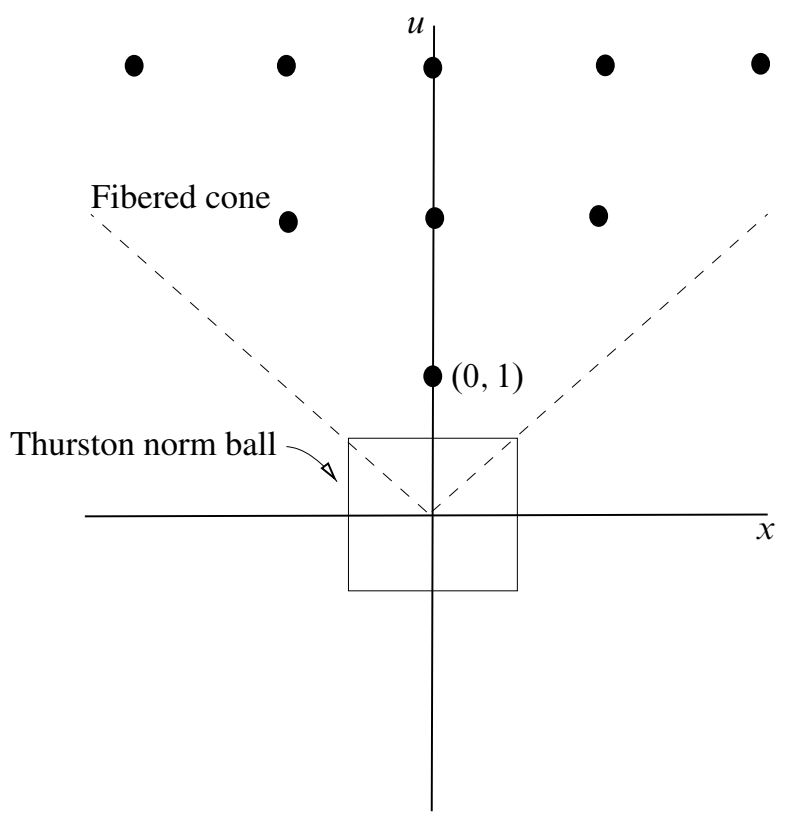

Figure 3: Fibered cone $\Psi$ containing $\psi=(0,1)$

Proposition 3.1 If $(a, b) \in \Psi_{\text {prim }}$, then the associated monodromy $\phi_{(a, b)}$ is pseudoAnosov with geometric dilatation given by

$$
\lambda_{(a, b)}=\left|\Theta_{L}\left(t^{a}, t^{b}\right)\right|=\left|t^{2 b}-t^{b}\left(1+t^{a}+t^{-a}\right)+1\right|,
$$

and homological dilatation given by

$$
\lambda_{(a, b)}^{\mathrm{hom}}=\left|\Delta_{L}\left(t^{a}, t^{b}\right)\right|=\left|t^{2 b}-t^{b}\left(1-t^{a}-t^{-a}\right)+1\right| .
$$

Corollary 3.2 If $(a, b) \in \Psi_{\text {prim }}$, then the associated monodromy $\phi_{(a, b)}$ is orientable if $a$ is odd and $b$ is even.

Proof If $a$ is odd and $b$ is even, then the roots of $\Theta_{L}\left(t^{a}, t^{b}\right)$ are the negatives of the roots of $\Delta_{L}\left(t^{a}, t^{b}\right)$. This implies that the geometric and homological dilatations of $\phi_{(a, b)}$ are equal, and therefore $\phi_{(a, b)}$ is orientable.

Later in this section, we prove the converse of Corollary 3.2. First we consider how the monodromy behaves near the boundary of $S_{(a, b)}$.

Proposition 3.3 Let $\phi_{(a, b)}: S_{(a, b)} \rightarrow S_{(a, b)}$ be the monodromy associated to $(a, b) \in$ $\Psi_{\text {prim }}$. The boundary components of $S_{(a, b)}$ has $\operatorname{gcd}(3, a)$ components coming from 
$T\left(K_{1}\right)$ and $\operatorname{gcd}(3, b)$ coming from $T\left(K_{2}\right)$. Thus, the total number of boundary components of $S_{(a, b)}$ is given by

$$
\left\{\begin{array}{l}
2 \quad \text { if } \operatorname{gcd}(3, a b)=1 \\
4 \quad \text { if } \operatorname{gcd}(3, a b)=3
\end{array}\right.
$$

Proof The number of components in $T\left(K_{i}\right) \cap S_{(a, b)}$ is the index of the image of $\pi_{1}\left(T\left(K_{i}\right)\right)$ in $\mathbb{Z}$ under the composition of maps

$$
\pi_{1}\left(T\left(K_{i}\right)\right) \rightarrow \pi_{1}(M) \rightarrow \mathbb{Z}
$$

induced by inclusion and $\psi_{(a, b)}$.

For $i=1,2$, let $\ell_{i}$ be the longitude of $K_{i}$ that is contractible in $S^{3} \backslash K_{i}$. Then, for $T\left(K_{1}\right)$ we have

$$
\psi_{(a, b)}\left(\mu_{1}\right)=a \quad \text { and } \quad \psi_{(a, b)}\left(\ell_{1}\right)=3 \psi_{(a, b)}\left(\mu_{2}\right)=3 b,
$$

so the number of boundary components contributed by $T\left(K_{1}\right)$ is

$$
\operatorname{gcd}(a, 3 b)=\operatorname{gcd}(3, a),
$$

since we are assuming that $\operatorname{gcd}(a, b)=1$. The contribution of $T\left(K_{2}\right)$ is computed similarly.

Proposition 3.4 The genus of $S_{(a, b)}$, for $(a, b) \in \Psi_{\text {prim }}$ is given by

$$
\begin{aligned}
g\left(S_{(a, b)}\right) & =|b|+\left(1-\frac{\operatorname{gcd}(3, a)+\operatorname{gcd}(3, b)}{2}\right) \\
& = \begin{cases}|b| & \text { if } \operatorname{gcd}(3, a b)=1 \\
|b|-1 & \text { if } \operatorname{gcd}(3, a b)=3 .\end{cases}
\end{aligned}
$$

Proof Equation (2) gives

$$
2|b|=\chi_{-}\left(S_{(a, b)}\right)=2 g-2+\operatorname{gcd}(3, a)+\operatorname{gcd}(3, b) .
$$

Proposition 3.5 Let $(a, b) \in \Psi_{\text {prim }}$, and let $\mathcal{F}$ be a $\phi_{(a, b)}$-invariant foliation. Then $\mathcal{F}$

(1) has no interior singularities,

(2) is $(3 b / \operatorname{gcd}(3, a))$-pronged at each of the $\operatorname{gcd}(3, a)$ boundary components coming from $T\left(K_{1}\right)$, and

(3) is $(b / \operatorname{gcd}(3, b))$-pronged at each of the $\operatorname{gcd}(3, b)$ boundary components coming from $T\left(K_{2}\right)$. 

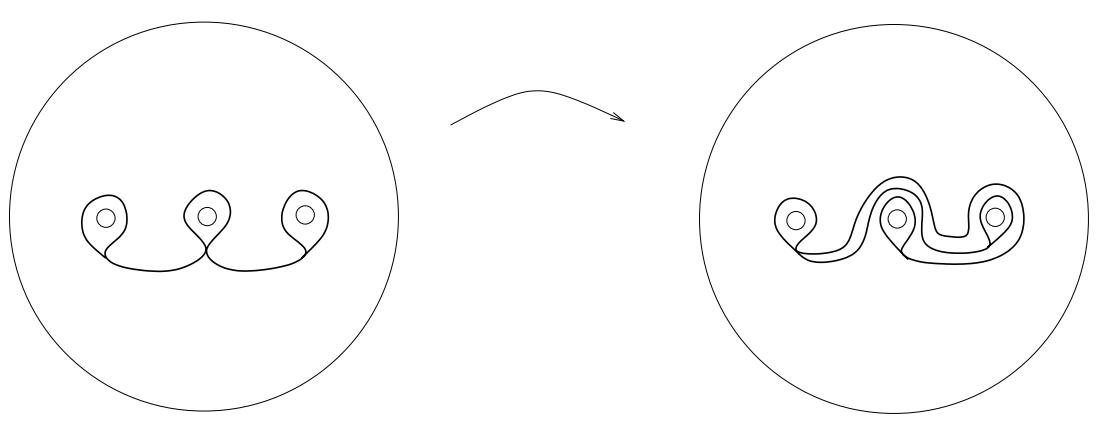

Figure 4: Train track for $\phi: S \rightarrow S$

Proof Let $\mathcal{L}$ be the lamination of $M$ defined by suspending $\mathcal{F}$ over $M$ considered as the mapping torus of $\phi$. From the train track for $\phi$ (Figure 4), one sees that each of the boundary components of $S$ are one-pronged, and that there are no other singularities. It follows that $\mathcal{L}$ has no singularities outside a neighborhood of the $K_{i}$, and near each $K_{i}$ the leaves of $\mathcal{L}$ come together at a simple closed curve $\gamma_{i} \in \mathrm{H}_{1}\left(T\left(K_{i}\right)\right)$. Write

$$
\gamma_{i}=r_{i} \mu_{i}+s_{i} \ell_{i}
$$

for $i=1,2$.

For $(a, b) \in \Psi_{\text {prim }}$, the number of intersections of $\gamma_{i}$ with $S_{(a, b)}$ is the image of $\gamma_{i}$ under the epimorphism

$$
\psi(a, b): \pi_{1}(M) \rightarrow \mathbb{Z}
$$

defining the fibration. Figure 4 shows that $s_{1}=1$ and $r_{2}=1$. Using the identities

$$
\begin{array}{ll}
s_{1}=1, & \ell_{1}=3 \mu_{2}, \\
r_{2}=1, & \ell_{2}=3 \mu_{1},
\end{array}
$$

we have

$$
\begin{aligned}
& \psi_{(a, b)}\left(\gamma_{1}\right)=r_{1} \psi_{n}\left(\mu_{1}\right)+3 \psi_{n}\left(\mu_{2}\right)=r_{1} a+3 b \\
& \psi_{(a, b)}\left(\gamma_{2}\right)=\psi_{n}\left(\mu_{2}\right)+3 s_{2} \psi_{n}\left(\mu_{1}\right)=3 s_{2} a+b .
\end{aligned}
$$

Let $m_{1}=\operatorname{gcd}(3, a)$ and $m_{2}=\operatorname{gcd}(3, b)$. Then $\phi_{(a, b)}$ is $\left(r_{1} a+3 b\right) / m_{1}$-pronged at $m_{1}$ boundary components and $\left(3 s_{2} a+b\right) / m_{2}$-pronged at $m_{2}$ boundary components. We find $r_{1}$ and $s_{2}$ by looking at some particular examples.

In general, if $f: \Sigma \rightarrow \Sigma$ is pseudo-Anosov on a compact oriented surface $\Sigma$ with genus $g$ and $n_{1}, \ldots, n_{k}$ are the number of prongs at the singularities and boundary 
components, then by the Poincaré-Hopf theorem

$$
\sum_{i=1}^{k}\left(n_{i}-2\right)=4 g-4 .
$$

For $(a, b)=(1, n), n$ not divisible by 3 , we have two singularities with number of prongs given by:

$$
\begin{aligned}
& \psi_{n}\left(\gamma_{1}\right)=r_{1}+3 n \\
& \psi_{n}\left(\gamma_{2}\right)=3 s_{2}+n .
\end{aligned}
$$

Plugging into (5) gives

$$
r_{1}+3 s_{2}=0 .
$$

The mapping class $\phi_{(1,2)}$ is the unique genus 2 pseudo-Anosov mapping class with dilatation equal to $\lambda_{2}$ (see Cho and Ham [4], and Lanneau and Thiffeault [16]) and has one 6-pronged singularity (see Hironaka and Kin [11]). Thus, $r_{1}=s_{2}=0$ and

$$
\gamma_{1}=\ell_{1}=3 \mu_{2} \quad \text { and } \quad \gamma_{2}=\mu_{2} .
$$

The claim follows.

Corollary 3.6 The map $\phi_{(a, b)}$ has singularities with number of prongs (or prong-type) given by

$$
\begin{cases}(3 b, b) & \text { if } \operatorname{gcd}(3, a b)=1 \\ (3 b, b / 3, b / 3, b / 3) & \text { if } \operatorname{gcd}(3, b)=3 \\ (b, b, b, b) & \text { if } \operatorname{gcd}(3, a)=3\end{cases}
$$

The degree of a singularity and the number of prongs differ by 2 , yielding Table 1 .

Corollary 3.7 If $b$ is odd, then $\phi_{(a, b)}$ is not orientable.

Corollary 3.8 For $(a, b) \in \Psi_{\text {prim }}, \phi_{(a, b)}$ is 1-pronged at one or more boundary components of $S_{(a, b)}$ if and only if $(a, b) \in\{(0,1),( \pm 1,3),( \pm 2,3)\}$.

Corollary 3.9 If $(a, b) \notin\{(0,1),( \pm 1,3),( \pm 2,3)\}$, then $\phi_{(a, b)}$ extends to the closure of $S_{(a, b)}$ over the boundary components to a mapping class $\bar{\phi}_{(a, b)}$ with the same dilatation as $\phi_{(a, b)}$.

Table 2 describes the pairs $(a, b) \in \Psi_{\text {prim }}$ that give rise to an orientable (or non-orientable) genus $g$ pseudo-Anosov mapping class. (Here $g \geq 4$.) 


\begin{tabular}{|c|l|l|}
\hline$g(\bmod 6)$ & orientable & non-orientable \\
\hline 0 & no example & $b=g+1, a=0(\bmod 3)$ \\
\hline 1 & $b=g+1, a=3(\bmod 6)$ & $b=g, a=1,2(\bmod 3)$ \\
\hline 2 & $b=g, a=1,5(\bmod 6)$ & $b=g+1, a=1,2(\bmod 3)$ \\
\hline 3 & $b=g+1, a=3(\bmod 6)$ & no example \\
\hline 4 & $b=g, a=1,5(\bmod 6)$ & $b=g+1, a=0(\bmod 3)$ \\
\hline 5 & $b=g+1, a=1,5(\bmod 6)$ & $b=g, a=1,2(\bmod 3)$ \\
\hline
\end{tabular}

Table 2: Fibrations of $M$ according to genus

\section{Minimal dilatations for the fibered face.}

Let $\Psi_{\text {prim }}$ be the primitive elements of the fibered cone discussed in Section 3. Let $d_{g}=\min \left\{\lambda(\psi): \psi \in \Psi_{\text {prim }}\right.$, genus of $\psi$ is $\left.g\right\}$, and $d_{g}^{+}=\min \left\{\lambda(\psi): \psi \in \Psi_{\text {prim }}\right.$, genus of $\psi$ is $g$, the monodromy of $\psi$ is orientable $\}$.

In this section, we finish the proofs of Theorem 1.4 and Theorem 1.5 and their consequences by determining $d_{g}$ and $d_{g}^{+}$.

Proposition 4.1 Let $(a, b) \in \Psi_{\text {prim. }}$. Then

$$
\lambda_{(a, b)}<\lambda_{\left(a^{\prime}, b^{\prime}\right)}
$$

if either

(1) $|a|<\left|a^{\prime}\right|$ and $|b|=\left|b^{\prime}\right|$; or

(2) $|a|=\left|a^{\prime}\right|$ and $|b|>\left|b^{\prime}\right|$.

Proof One compares the slopes of rays from the origin to $(a, b)$ and $\left(a^{\prime}, b^{\prime}\right)$. The claim follows from Theorem 2.2.

Proposition 4.2 For $b \geq 3$, we have

$$
\lambda_{(1, b)} \geq \lambda_{(3, b+1)}
$$

with equality when $b=3$. 
Proof Let $\lambda=\lambda_{(3, b+1)}$. We will show that $\operatorname{LT}_{(1, b)}(\lambda)<0$. Multiplying by $\lambda^{2}$ and using the fact that $\operatorname{LT}_{(3, b+1)}(\lambda)=0$ gives

$$
\begin{aligned}
\lambda^{2} L T_{(1, b)}(\lambda) & =\lambda^{2} L T_{(1, b)}(\lambda)-L T_{(3, b+1)}(\lambda) \\
& =\lambda^{b+4}-\lambda^{b+3}-\lambda^{b+2}+\lambda^{b-2}+\lambda^{2}-1 \\
& =(\lambda-1)\left(\lambda^{b+3}-\lambda^{b-2}\left(\lambda^{3}+\lambda^{2}+\lambda+1\right)+\lambda+1\right) \\
& =(\lambda-1) \lambda^{b-2}\left[\lambda^{5}-\lambda^{3}-\lambda^{2}-\lambda-1+\lambda^{2-b}(\lambda+1)\right] .
\end{aligned}
$$

Thus, it is enough to show that for $\lambda>1$ and $b>3$

$$
\lambda^{5}-\lambda^{3}-\lambda^{2}-\lambda-1+\lambda^{2-b}(\lambda+1)<0 .
$$

Let $C$ be the quantity on the left side of this inequality. Then

$$
C<\lambda^{5}-\lambda^{3}-\lambda^{2}=\lambda^{2}\left(\lambda^{3}-\lambda-1\right) .
$$

One can check that the right hand side is negative for

$$
1<\lambda<1.3
$$

By Proposition 4.1, $\lambda$ decreases as $b$ increases. A check shows that

$$
1<\lambda_{(3,5)}<1.3
$$

and hence $C<0$ for $b \geq 4$. For $b=3$, one checks directly that

$$
\lambda_{(1,3)}=\lambda_{(3,4)} \text {. }
$$

Remark The mapping class $\phi_{(1,3)}$ is defined on a genus 2 surface with four boundary components, with prong-type $(3,1,1,1)$ and is not orientable. The mapping class $\phi_{(3,4)}$ is defined on a genus 3 surface with prong-type $(4,4,4,4)$ and is orientable. By Proposition 4.2 these two examples have the same dilatation.

Proposition 4.1 and Proposition 4.2 imply the following.

Proposition 4.3 The sequences $\lambda_{(1, b)}$ and $\lambda_{(3, b)}$ satisfy:

$$
\lambda_{(1, b)}>\lambda_{(3, b+1)}>\lambda_{(1, b+1)} .
$$

Table 3 describes the pairs $(a, b) \in \Psi_{\text {prim }}$ that give rise to the minima $d_{g}$ and $d_{g}^{+}$ realized on $M$. 


\begin{tabular}{|c|l|l|}
\hline$g \bmod 6$ & $\lambda\left(\phi_{(a, b)}\right)=d_{g}^{+}, \phi_{(a, b)}$ orientable & $\lambda\left(\phi_{(a, b)}\right)=d_{g}$ \\
\hline 0 & no example & $(3, g+1)$ \\
\hline 1 & $(3, g+1)$ & $(3, g+1)$ \\
\hline 2 & $(1, g)$ & $(1, g+1)$ \\
\hline 3 & $(3, g+1)$ & $(3, g+1)$ \\
\hline 4 & $(1, g)$ & $(3, g+1)$ \\
\hline 5 & $(1, g+1)$ & $(1, g+1)$ \\
\hline
\end{tabular}

Table 3: Pairs $(a, b)$ giving smallest dilatations for $\phi \in \Phi\left(M_{\mathrm{sb}}\right)$

Proposition 4.4 For $n \geq 2$,

$$
\lim _{n \rightarrow \infty}\left(\lambda_{(a, n)}\right)^{n}=\frac{3+\sqrt{5}}{2},
$$

for any fixed $a$.

Proof The rays through the lattice points $(a, n) \in \Lambda_{M}$ on the fibered face of $\psi$ converge to the ray through $(0,1)$.

Corollary 4.5 For the minimal dilatations $d_{g}$ and $d_{g}^{+}$that are realized on $M$, we have

$$
\lim _{g \rightarrow \infty}\left(d_{g}\right)^{g}=\frac{3+\sqrt{5}}{2}, \quad \text { and } \quad \lim _{\substack{g \rightarrow \infty \\ g \neq 0(\bmod 6)}}\left(d_{g}^{+}\right)^{g}=\frac{3+\sqrt{5}}{2} .
$$

Table 3 and Corollary 3.9 complete the proofs of Theorem 1.4 and Theorem 1.5. A pictorial view of how the elements of $\Psi$ giving the least dilatations for each genus up to 12 lie on a fibered cone of $M$ is shown in Figure 5.

The results of this paper and those in Aaber and Dunfield [1], Kin and Takasawa [14], and Lanneau and Thiffeault [16] imply that for genus $g=2,3,4,5,7$, and 8 ,

$$
\delta_{g}^{+}=\lambda(a, b)
$$

where

$$
(a, b)= \begin{cases}(1, g) & \text { if } g=2,3,4 \text { or } 8 \\ (1, g+1) & \text { if } g=5 \\ (2, g+2) & \text { if } g=7\end{cases}
$$

and

$$
\delta_{6}^{+} \geq \lambda(1,6)
$$

These results suggest the following generalization to Question 1.3. 
13

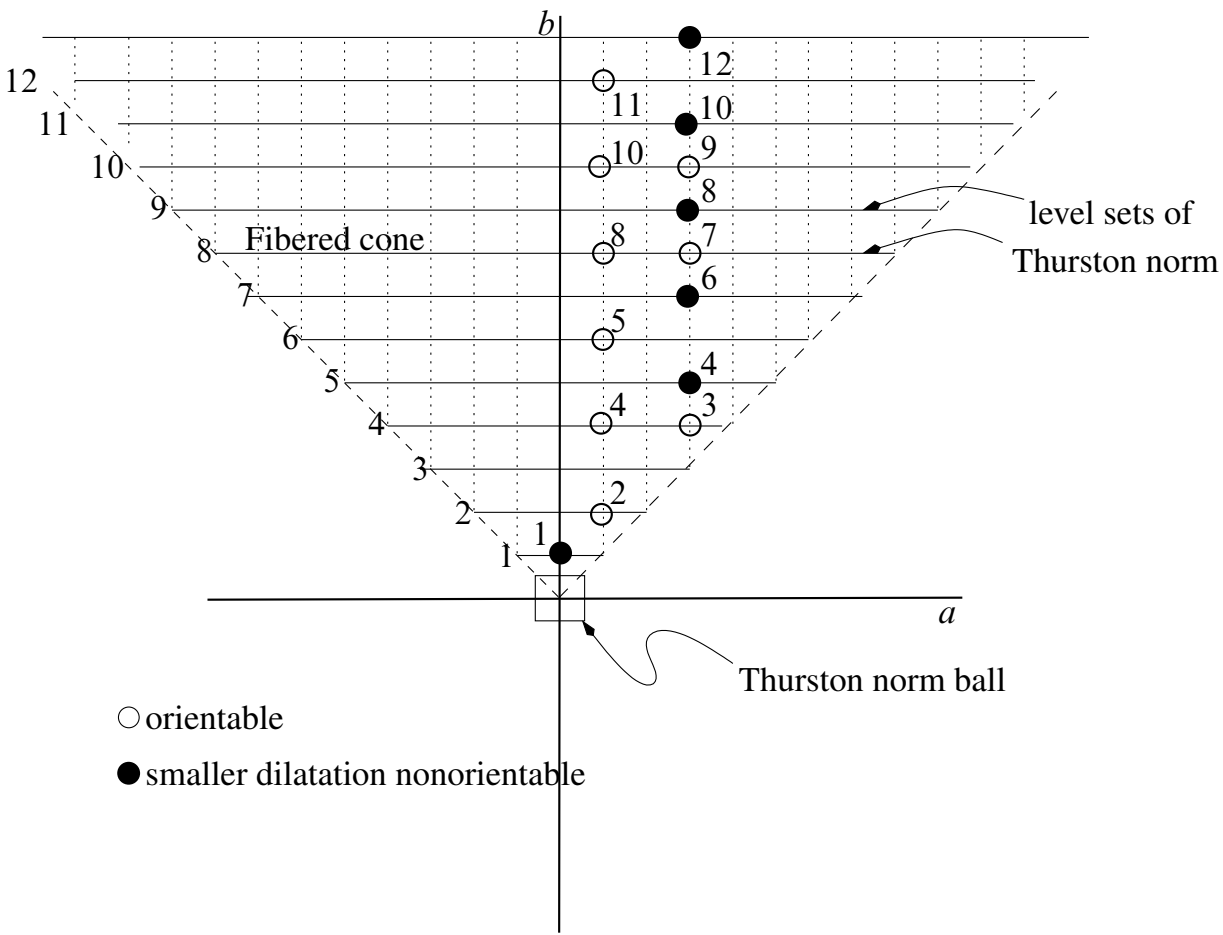

Figure 5: Minima for $d_{g}$ and $d_{g}^{+}$in genus $g=1, \ldots, 12$

Question 4.6 For every $g \geq 2$, is it true that

$$
\delta_{g}^{+}=\lambda_{(a, b)}
$$

for some $a, b$ with $b \geq g \geq a \geq 1$ ?

\section{References}

[1] J Aaber, N Dunfield, Closed surface bundles of least volume, preprint (2010)

[2] P Arnoux, J-C Yoccoz, Construction de difféomorphismes pseudo-Anosov, C. R. Acad. Sci. Paris Sér. I Math. 292 (1981) 75-78 MR610152

[3] A J Casson, S A Bleiler, Automorphisms of surfaces after Nielsen and Thurston, London Mathematical Society Student Texts 9, Cambridge University Press, Cambridge (1988) MR964685

[4] J-H Cho, J-Y Ham, The minimal dilatation of a genus-two surface, Experiment. Math. 17 (2008) 257-267 MR2455699 
[5] B Farb, Some problems on mapping class groups and moduli space, from: "Problems on mapping class groups and related topics", Proc. Sympos. Pure Math. 74, Amer. Math. Soc., Providence, RI (2006) 11-55 MR2264130

[6] B Farb, C Leininger, D Margalit, Small dilatation pseudo-Anosovs and 3-manifolds, preprint (2009)

[7] A Fathi, F Laudenbach, V Poenaru, Travaux de Thurston sur les surfaces, Astérisque 66, Société Mathématique de France, Paris (1979) MR568308 Séminaire Orsay, With an English summary

[8] D Fried, Flow equivalence, hyperbolic systems and a new zeta function for flows, Comment. Math. Helv. 57 (1982) 237-259 MR684116

[9] E Hironaka, The Lehmer polynomial and pretzel links, Canad. Math. Bull. 44 (2001) 440-451 MR1863636

[10] E Hironaka, Chord diagrams and Coxeter links, J. London Math. Soc. (2) 69 (2004) 243-257 MR2025339

[11] E Hironaka, E Kin, A family of pseudo-Anosov braids with small dilatation, Algebr. Geom. Topol. 6 (2006) 699-738 MR2240913

[12] N V Ivanov, Coefficients of expansion of pseudo-Anosov homeomorphisms, Zap. Nauchn. Sem. Leningrad. Otdel. Mat. Inst. Steklov. (LOMI) 167 (1988) 111-116, 191 MR964259

[13] E Kin, M Takasawa, An asymptotic behavior of the dilatation for a family of pseudoAnosov braids, Kodai Math. J. 31 (2008) 92-112 MR2414236

[14] E Kin, M Takasawa, Pseudo-Anosovs on closed surfaces having small entropy and the Whitehead sister link exterior, preprint (2010)

[15] T Koberda, A Silberstein, Representations of Galois groups on the homology of surfaces, preprint (2009)

[16] E Lanneau, J-L Thiffeault, On the minimum dilatation of pseudo-Anosov homeomorphisms on surfaces of small genus, Ann. Inst. Fourier (to appear)

[17] D H Lehmer, Factorization of certain cyclotomic functions, Ann. of Math. (2) 34 (1933) 461-479 MR1503118

[18] C J Leininger, On groups generated by two positive multi-twists: Teichmüller curves and Lehmer's number, Geom. Topol. 8 (2004) 1301-1359 MR2119298

[19] B Martelli, C Petronio, Dehn filling of the "magic" 3-manifold, Comm. Anal. Geom. 14 (2006) 969-1026 MR2287152

[20] C T McMullen, Polynomial invariants for fibered 3-manifolds and Teichmüller geodesics for foliations, Ann. Sci. École Norm. Sup. (4) 33 (2000) 519-560 MR1832823 
[21] C T McMullen, The Alexander polynomial of a 3-manifold and the Thurston norm on cohomology, Ann. Sci. École Norm. Sup. (4) 35 (2002) 153-171 MR1914929

[22] H Minakawa, Examples of pseudo-Anosov homeomorphisms with small dilatations, J. Math. Sci. Univ. Tokyo 13 (2006) 95-111 MR2277516

[23] R C Penner, Bounds on least dilatations, Proc. Amer. Math. Soc. 113 (1991) 443-450 MR1068128

[24] D Rolfsen, Knots and links, Mathematics Lecture Series 7, Publish or Perish, Berkeley, CA (1976) MR0515288

[25] W P Thurston, A norm for the homology of 3-manifolds, Mem. Amer. Math. Soc. 59 (1986) i-vi and 99-130 MR823443

[26] W P Thurston, On the geometry and dynamics of diffeomorphisms of surfaces, Bull. Amer. Math. Soc. (N.S.) 19 (1988) 417-431 MR956596

[27] A Y Zhirov, On the minimum dilation of pseudo-Anosov diffeomorphisms of a double torus, Uspekhi Mat. Nauk 50 (1995) 197-198 MR1331364

Department of Mathematics, Florida State University

Tallahasse FL 32301, USA

hironaka@math.fsu.edu

Received: 29 October 2009 Revised: 16 April 2010 